\title{
AVALIAÇÃO DO DESPERDÍCIO DE ALIMENTOS NA MORADIA ESTUDANTIL DA FCT/UNESP E DO INTERESSE NA SUA REDUÇÃO
}

\section{FOOD WASTE ASSESSMENT OF STUDENT HOUSING IN FCT / UNESP AND INTEREST IN YOUR REDUCTION}

Elelan Vitor Machado ${ }^{1 *}$, Maricielo Well Quispe Núnez, Maria Cristina Rizk

Universidade Estadual Paulista - FCT/UNESP, Curso de Engenharia Ambiental, Presidente Prudente, SP. e-mail: : elelan machado@outlook.com. * Bolsista PROEX/UNESP

RESUMO - O desperdício de alimentos é um problema amplamente discutido, sendo muito importante a busca pela sua redução. Nesse sentido, o presente trabalho teve como objetivo avaliar o desperdício de alimentos nas cozinhas coletivas da Moradia Estudantil da FCT/UNESP e o interesse dos moradores na sua redução. Foram avaliados três fatores de desperdícios de alimentos: fator de correção, sobras totais e restos, além da aplicação de questionários relacionados ao desperdício de alimentos. Os resultados indicaram que a porcentagem média de sobras por dia foi de $33,4 \%$ e a de restos foi de $67,6 \%$. O fator de correção médio obtido no pré-preparo dos alimentos de origem de hortifrúti foi de 1,313. Segundo o questionário aplicado aos integrantes da Moradia Estudantil, cerca do $80 \%$ dos alunos acha importante a implantação de técnicas de minimização de resíduos sólidos e $100 \%$ mostrou interesse em saber a quantidade de desperdício gerado e quantas pessoas poderiam ser alimentadas com este desperdício.

Palavras-chave: desperdício de alimento; educação ambiental; resíduos sólidos.

ABSTRACT - Food waste is a widely discussed issue, being very important its reduction. In this sense, the present work aimed to evaluate the food waste in the collective kitchens of the Student's Housing of FCT/UNESP and the interest of the residents in its reduction. Three factors of food waste were evaluated: the correction factor, total leftover and rests. Also, it had been applied to the students questionnaires about the food waste. The results indicated that the leftovers percentage average per day was $33.4 \%$ and the rests was $67.6 \%$. The medium correction factor obtained in pre-preparing of vegetables was 1.313. According to the questionnaire administered to members of the Student's Housing about $80 \%$ of the students thought it is important the implementation of techniques for the solid waste reduction and $100 \%$ showed interesting to know the amount of waste generated and how many people could be fed with this waste.

Keywords: food waste; environmental education; solid waste. 


\section{INTRODUÇÃO}

A fome e o desperdício de alimentos são problemas que o Brasil enfrenta, constituindo-se em um dos paradoxos do nosso país que é um dos maiores exportadores mundiais de alimentos, e também é um dos campeões de desperdício (MARCHETTO et al., 2008).

O desperdício de alimentos é um problema amplamente discutido e que está incorporado à cultura brasileira, sendo movido por causas econômicas, políticas, culturais e tecnológicas, que abrangem as principais etapas da cadeia de movimentação: produção, transporte, comercialização, sistema de embalagem e armazenamento (SILVÉRIO e OLTRAMARI, 2014).

Do total de desperdício no país, $10 \%$ ocorrem durante a colheita; $50 \%$ no manuseio e transporte dos alimentos; $30 \%$ nas centrais de abastecimento, e os últimos $10 \%$ ficam diluídos entre supermercados e consumidores. Não há estudos conclusivos que determinem o desperdício nas casas e nos restaurantes, mas estima-se que a perda no setor de refeições coletivas chegue a $15 \%$ e, nas nossas cozinhas, a 20\% (MARCHETTO et al., 2008).

Zotesso (2013) cita que do ponto de vista social, o desperdício de alimentos representa uma enorme contradição, uma vez que mais de um bilhão de pessoas passam fome no mundo; do ponto de vista ambiental, representa a desnecessária extração de recursos da natureza e a poluição ambiental; e do ponto de vista econômico, representa um fator de elevação do custo.

Nesse cenário, uma das soluções apontadas é a transformação do modo de consumir, permitindo que os cidadãos tomem as decisões acertadas em seus atos de consumo, adquirindo consciência do impacto coletivo, ambiental e social em que se traduzem suas opções individuais de consumo para a promoção da qualidade de vida e do desenvolvimento local (COSTA e TEODÓSIO, 2011).

Dentro deste contexto, o presente estudo teve como objetivo analisar o desperdício de alimentos no sistema de alimentação coletiva da Moradia Estudantil da FCT/UNESP, bem como avaliar o interesse dos integrantes em reduzir este desperdício.

\section{METODOLOGIA}

Neste estudo, foram avaliados três fatores de desperdício de alimentos que ocorrem nas cozinhas coletivas da Moradia Estudantil da FCT/UNESP, os quais são: fator de correção, porcentagem de sobras totais e índice de restos.

A partir destes fatores foi possível determinar o consumo per capita por 
refeição e o número de pessoas que poderiam ser alimentadas com o desperdício.

O fator de correção prevê as perdas que ocorrem na etapa de pré-preparo dos alimentos de origem de hortifrúti, como perdas oriundas da limpeza dos alimentos, remoção das cascas, sementes, dentre outros. Este fator é uma constante para um determinado alimento de mesma qualidade, onde é representado pela razão de seu peso bruto e o peso líquido (ORNELLAS, 2007).

Desse modo, os alimentos foram pesados antes e após a sua manipulação (pré-preparo). O fator de correção foi calculado de acordo coma equação 1 (ABREU et al., 2009). O fator de correção foi determinado pela média dos fatores de correção de todos os hortifrúti frequentemente consumidos na Moradia Estudantil.

Fator de Correção $(F C)=\frac{\text { Peso Bruto }(\mathrm{PB})}{\text { Peso Líquido(PL) }}(1)$

O percentual de sobras totais foi determinado pela razão entre o peso das sobras (PS) e o peso total dos alimentos produzidos (PTP), conforme a equação 2 (ABREU et al., 2009).

$\%$ Sobras Totais $=\frac{\text { Peso Sobras Totais }(\text { PST })}{\text { Peso Total Produzido }(\text { PTP })} \times 100$ (2)

O índice de restos foi calculado de acordo com a equação 3 (ABREU et al., 2009). Índice de Resto(IR) $=\frac{\text { Peso da Refeição Rejeitada(PR) }}{\text { Peso da Refeição Servida(PRS) }} \times 100$ (3)

Para o cálculo de quantas pessoas que poderiam ser alimentadas com os restos de alimentos do dia, foi determinado consumo per capita, segundo a equação 4 (SALES, 2009).

Consumo per capita por refeição $(\mathrm{kg})=\frac{\text { Peso da Refeição Servida }}{\text { Número de Refeições Servidas }}$ (4)

Também, foi determinado o número de pessoas que poderiam ser alimentadas por dia com os restos produzidos, segundo a equação 5 .
Além dos fatores de desperdício, foi avaliado, por meio da aplicação de questionários a 10 integrantes da Moradia
Estudantil, o interesse dos alunos a respeito do desperdício de alimentos e da implantação de técnicas para diminuição ou 


\section{Questionário aplicado aos integrantes do sistema de alimentação coletiva da Moradia Estudantil}

1 - Segundo as suas observações, existe desperdício de alimentos no sistema de alimentação coletiva, tanto no preparo das refeições, quanto nas sobras dos pratos?

( ) 1-2 (não há) ( ) 3-4 (baixo) ( ) 5-6 (médio) ( ) 7-8 (alto) ( ) 9-10 (muito alto)

2 - Você gostaria de saber qual a quantidade de desperdício gerado pelos integrantes do sistema de alimentação coletiva, tais como, sobras, restos e quantas pessoas que poderiam ser alimentadas com esse desperdício?

( ) Sim ( ) Não

3 - Qual o seu interesse na implantação de técnicas no sistema de alimentação coletiva, visando a minimização da geração dos resíduos sólidos?

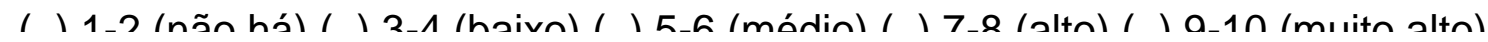

Figura 1. Questionário aplicado aos integrantes da Moradia Estudantil

\section{RESULTADOS}

Os resultados obtidos no cálculo das

equações de 1 a 5 , apresentadas na

metodologia, estão na Tabela 1.

Tabela 1. Desperdício de alimentos no sistema de alimentação coletiva da Moradia Estudantil

\begin{tabular}{lc}
\hline \multicolumn{1}{c}{ Parâmetros avaliados } & Resultados por dia \\
\hline Fator de correção médio & 1,313 \\
\hline \% de Sobras Totais & $33,4 \%$ \\
\hline Índice de Restos & $67,6 \%$ \\
\hline Desperdício total & $9,742 \mathrm{~kg}$ \\
\hline Desperdício per capita & $0,083 \mathrm{~kg}$ \\
\hline $\begin{array}{l}\text { Número de pessoas que poderiam ser } \\
\text { alimentadas com o desperdício }\end{array}$ & 19,410 pessoas \\
\hline
\end{tabular}

As Figuras de 2 a 4 apresentam os resultados obtidos na aplicação do questionário aos integrantes da Moradia Estudantil. 


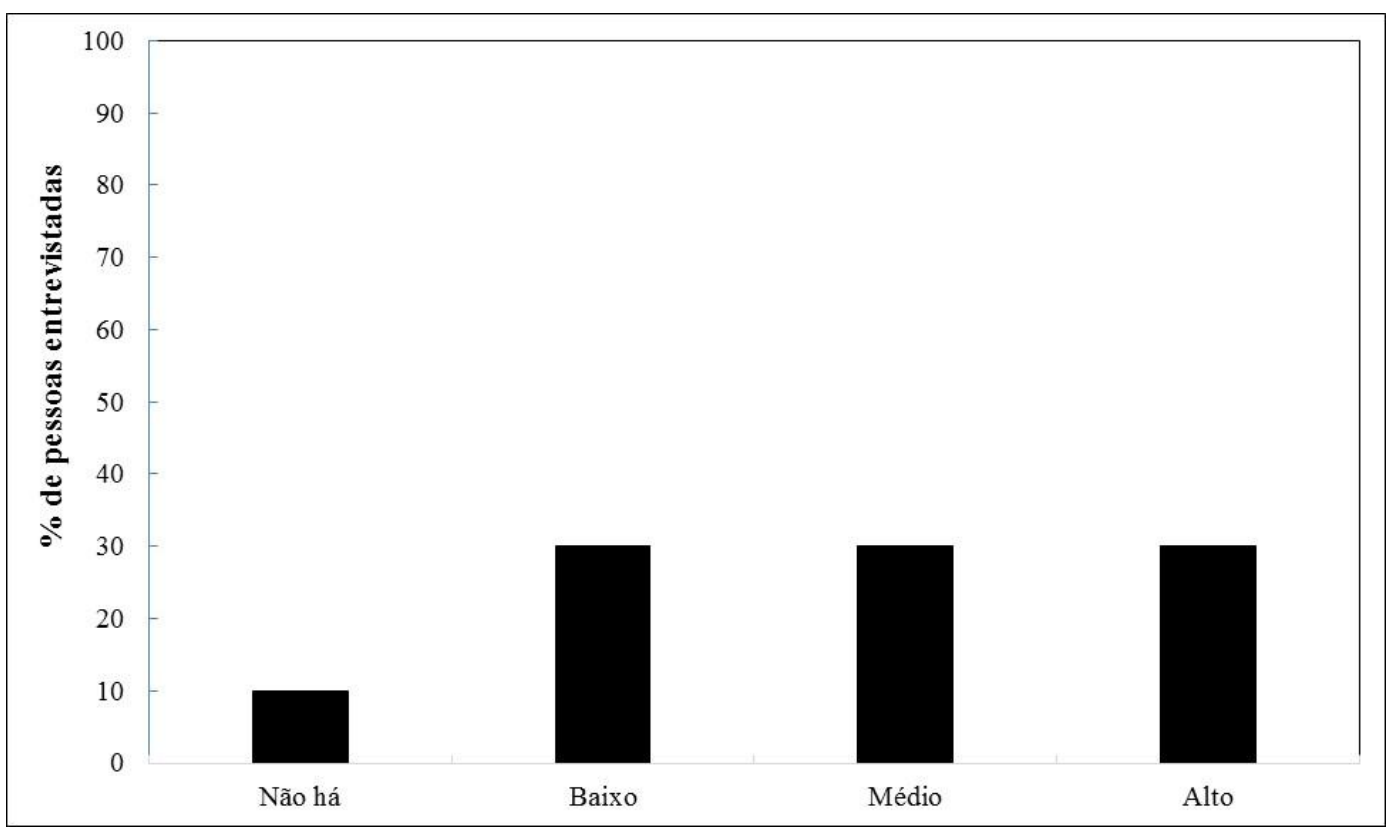

Figura 2. Conhecimento da quantidade de desperdício de alimentos gerada na Moradia Estudantil

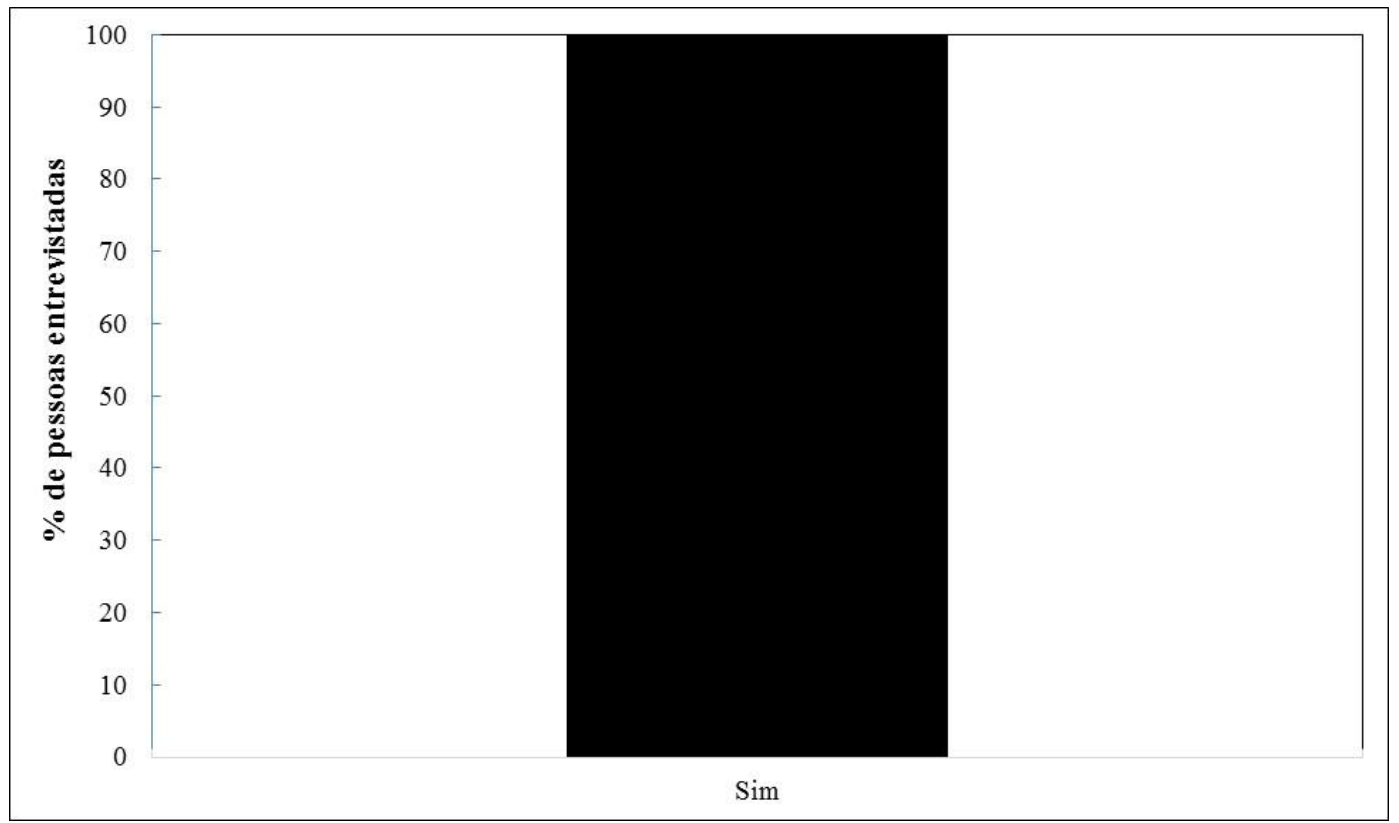

Figura 3. Interesse em conhecer a quantidade real do desperdício de alimentos na Moradia Estudantil 


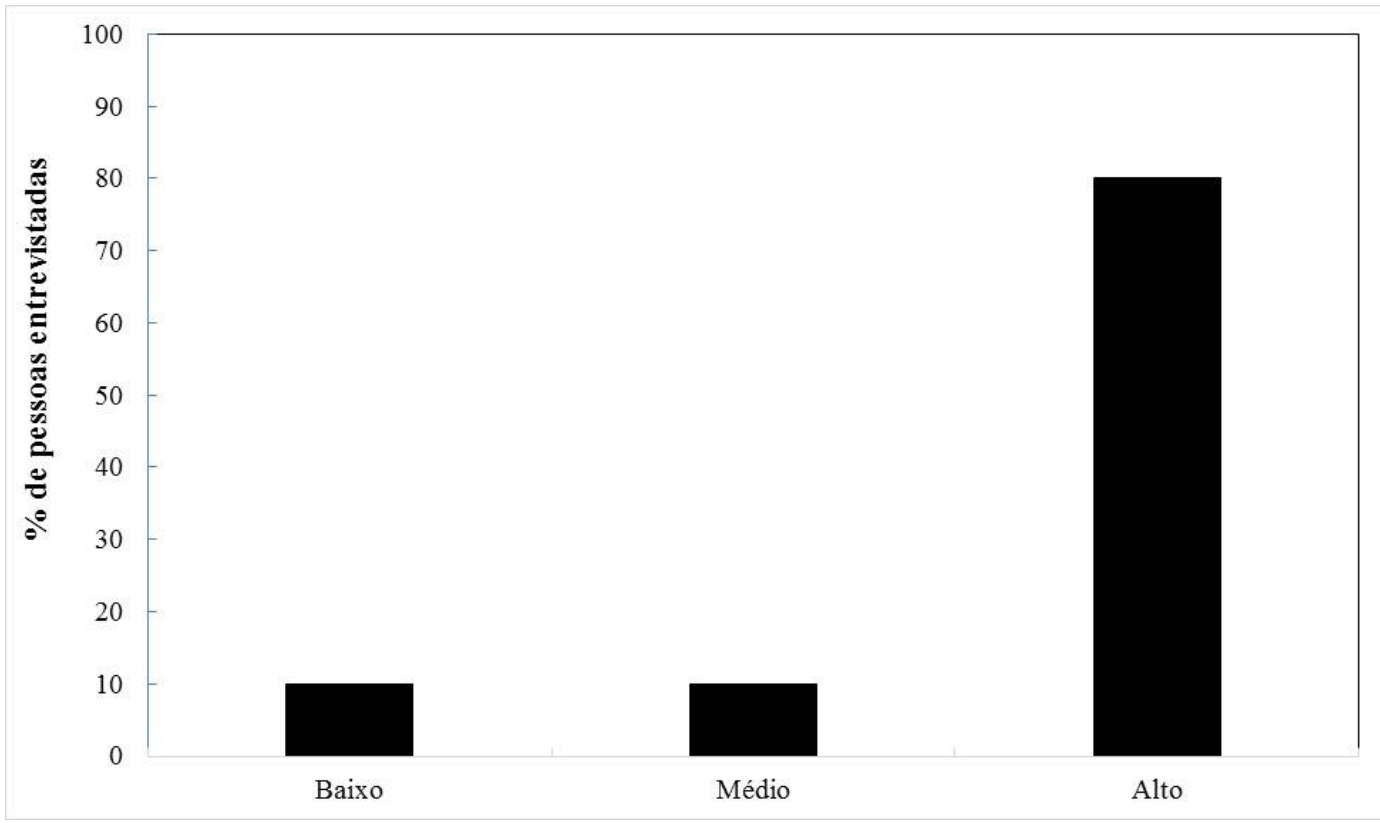

Figura 4. Interesse na implantação de técnicas de minimização do desperdício de alimentos na Moradia Estudantil

\section{DISCUSSÃO}

De acordo com a Tabela 1, o desperdício de alimentos nas cozinhas coletivas da Moradia Estudantil foi significativo, isto ocorre geralmente pela falta de comunicação entre os integrantes da Moradia, que não informam previamente se irão se alimentar no sistema coletivo ou não, fazendo com que seja feita uma quantidade maior de refeições, que talvez não serão consumidas.

O fator de correção médio, referente as perdas que ocorrem na etapa de prépreparo dos alimentos de origem de hortifrúti, foi de 1,313, o que possibilita dizer que parte do desperdício se deve provavelmente a falhas nos processos de compra e cortes e/ou retirada excessiva de cascas durante a manipulação dos alimentos.
Estes procedimentos podem influenciar na quantidade total produzida, ocasionando aumento desnecessário do preço a pagar mensalmente nos sistemas de alimentação coletiva, além da maior geração de resíduos.

Observou-se que a porcentagem de sobras por dia foi de $33,4 \%$ aproximadamente, este valor indica que quase a $1 / 3$ dos alimentos preparados por dia não chega a ser servido nos pratos. Uma das causas pode ser o descuido do cozinheiro em deixar os alimentos no fogo por mais tempo que o devido, o que faz com que parte dos alimentos se perca no fundo das panelas.

$O$ índice de restos gerado foi de $67,6 \%$, evidenciando que este foi o principal contribuinte para o elevado valor no desperdício. 0 índice de restos se deve ao excesso de alimentos servidos nos pratos por 
refeição, que acabam não sendo consumidos pelos usuários do sistema de alimentação coletiva.

O desperdício total diário foi de 9,742 kg (soma das sobras e dos restos diários gerados nos sistemas de alimentação coletiva) e o desperdício per capita diário foi de $0,083 \mathrm{~kg}$. O número de pessoas que poderiam ser alimentadas com o desperdício é de 19,410 pessoas/dia. Isto significa que 38,8 refeições por dia são desperdiçadas, o que indica a necessidade da adoção de técnicas adequadas para a melhoria do sistema de alimentação coletiva.

Em relação aos questionários aplicados, a Figura 2 indicou que 10\% dos moradores acredita que não há desperdício de alimentos, 30\% avaliou o desperdício como baixo, $30 \%$ como médio e $30 \%$ como alto. Isso mostra que existem dúvidas, por parte dos integrantes do sistema de alimentação coletiva, a respeito de como se dá o desperdício de alimentos e da sua percepção na identificação/quantificação do mesmo.

A Figura 3 mostrou que todos os entrevistados consideraram importante saber a quantidade de alimento que é desperdiçada nos sistemas de alimentação coletiva. Este fato revela que há uma sensibilização imediata por parte dos moradores a respeito do desperdício de alimentos e uma preocupação sobre o tema.
$\mathrm{Na}$ Figura 4 foi observado que a maioria dos alunos tem interesse em aprender técnicas para diminuir o desperdício de alimentos e, consequentemente, a geração de resíduos sólidos. Estes resultados indicaram que há uma preocupação social e ambiental em relação aos alimentos que poderiam ser consumidos de maneira mais adequada.

Assim, os resultados encontrados no presente estudo demonstram a necessidade de revisão no comportamento alimentar dos integrantes dos sistemas de alimentação coletiva da Moradia Estudantil e de uma conscientização sobre o desperdício, que poderia influenciar nas práticas alimentares dos comensais.

\section{CONCLUSÃO}

De acordo com os resultados deste trabalho foi possível constatar que há desperdícios significativos e com o desperdício diário seria possível alimentar aproximadamente 19 pessoas.

A respeito dos questionários aplicados aos moradores, há $100 \%$ de interesse em conhecer a quantidade de desperdício gerado pelos mesmos e há o reconhecimento de que é necessário implantar técnicas de minimização de resíduos sólidos.

Portanto, o presente trabalho mostrou que é necessário adotar técnicas de consumo consciente, minimizando o 
desperdício de alimentos, bem como a geração de resíduos sólidos.

\section{AGRADECIMENTOS}

Os autores agradecem ao Programa Institucional de Bolsas de Iniciação Científica (PIBIC) e a Pró-Reitoria de Extensão Universitária (PROEX) da Universidade Estadual Paulista "Júlio de Mesquita Filho" (UNESP) pelo apoio financeiro.

\section{REFERÊNCIAS}

ABREU, E. S. de; SPINELLI, M. G. N.; PINTO, A. M. S. Gestão de unidades de alimentação e nutrição: um modo de fazer.3 ed. São Paulo: Metha, 2009.

COSTA, D. V.; TEODÓSIO, A. S.

Desenvolvimento sustentável, consumo e cidadania: um estudo sobre a (des)articulação da comunicação de organizações da sociedade civil, do estado e das empresas. RAM, Revista de Administração Mackenzie. São Paulo, 2011, v. 12, n. 3, p. 114-145.

MARCHETTO, A. M. P., ATAIDE, H. K., MASSON, M. L. F., et al., Avaliação das partes desperdiçadas de alimentos no setor de hortifrúti visando seu reaproveitamento. Revista Simbio-Logia. São Paulo, 2008, v.1, n.2, p. 1-14.

ORNELLAS, L. H. Técnica dietética: seleção e preparo de alimentos. 8. ed. São Paulo:

Atheneu, 2007.

SALES, P. G. L. Diagnóstico da geração de resíduos sólidos em Restaurantes públicos populares do município do Rio de Janeiro: contribuição para minimização de desperdícios. Dissertação de Pós-graduação em Nutrição, Instituto de Nutrição Josué de
Castro, Universidade Federal do Rio de Janeiro. Rio de Janeiro, 2009.

SILVÉRIO, G. A., OLTRAMARI, K. Desperdício de alimentos em Unidades de Alimentação e Nutrição brasileiras. Ambiência - Revista do Setor de Ciências Agrárias e Ambientais. São Paulo, 2014, v. 10, n. 1, p. 125-133.

ZOTESSO, J. P. Diagnóstico da situação e caracterização dos resíduos sólidos gerados no restaurante universitário da universidade estadual de Maringá. Dissertação de mestrado em Engenharia Química, Universidade Estadual de Maringá. Maringá, 2013. 